\title{
Jordan-Wigner Transformation in Higher Dimensions
}

\section{Exact bosonization in two spatial dimensions and a new class of lattice gauge theories}

Authors: Y.-A. Chen, A. Kapustin, and D. Radičević

Annals of Physics 393, 234 (2018)

2. Constraints of kinematic bosonization in two and higher dimensions

Authors: A. Bochniak, B. Ruba, J. Wosiek, and A. Wyrzykowski

Phys. Rev. D 102, 114502 (2020)

\section{Symmetric Jordan-Wigner transformation in higher dimensions}

Authors: H.-C. Po

arXiv:2107.10842

\section{Recommended with a Commentary by Masaki Oshikawa, Institute for Solid State Physics, University of Tokyo}

Particle statistics is one of the most fascinating aspects of quantum mechanics. While the Bose-Einstein statistics is already counter-intuitive, bosons may be understood as elementary excitations of quantized classical fields. Fermions, on the other hand, do not seem to have any classical counterpart. Formally they arise by quantizing Grassmann fields, but this may not be too helpful for intuitive understanding. Yet, fermions are allowed within principles of quantum mechanics, and indeed they are important building blocks of our universe. Not only the electrons, but also the "matter fields" in the standard model of elementary particles are fermionic. Fermi-Dirac statistics is indeed important for the stability of the matter. On the other hand, Fermi-Dirac statistics also poses computational challenge. The notorious negative sign problem in Quantum Monte Carlo simulations is a consequence of the Fermi-Dirac statistics (although the sign problem can appear also in bosonic systems). The computational difficulty of fermions has also become a renewed issue [1], as quantum computers will be based on bosonic qubits.

Given the fundamental difference between bosons and fermions, it is remarkable that a mapping between a fermionic system and a bosonic system, known as Jordan-Wigner transformation [2], exists in one spatial dimension. The mapping can be defined exactly for a one-dimensional lattice, as discussed in Ref. [3]. The exact solution of the quantum $S=1 / 2$ chain, which follows from the exact Jordan-Wigner transformation, is an important basis to understand quantum magnetism in one dimension. It is then a natural desire to extend the 
Jordan-Wigner transformation to higher dimensions, and many attempts have been made. In particular, the "statistical transmutation" by a flux attachment [4] has become a popular picture to study two-dimensional systems, especially Quantum Hall states. While it is a useful formulation, the conventional flux attachment argument does not provide an exact mapping for lattice models (and does not work in $3+1$ and higher dimensions). The difficulty of extending the Jordan-Wigner transformation to higher dimensions can be naturally understood by recalling the standard one-dimensional construction. The fermionic creation operator is mapped to the bosonic one with the "Jordan-Wigner string" which extends to the infinity. The anticommutativity between the creation operator and the Jordan-Wigner string ensures the fermionic anticommutation relation. Although the Jordan-Wigner transformation is non-local, it maps many of the physical models such as the fermionic tight-binding model with the nearest neighbor hoppings on the one-dimensional chain to local bosonic model such as the $S=1 / 2 \mathrm{XY}$ chain. This is because most of the Jordan-Wigner string of the two operators in the hopping term cancel with each other. The Jordan-Wigner transformation is useful because of this locality in both sides of the mapping. A direct application of the one-dimensional Jordan-Wigner transformation to higher dimensional systems is possible by introducing a sequential numbering of all the sites. However, it is not quite satisfactory, as the short-range hoppings are mapped to terms with long Jordan-Wigner strings remaining uncancelled. We would like to have a transformation which maps local Hamiltonian (with only short-range hoppings) to a local Hamiltonian. In fact, such transformations have been developed for higher dimensions, and recently there is a renewed interest in the subject as shown in the highlighted papers and their sequels.

In retrospect, a hint for the extension can already be found in the classical Ising model in two dimensions. It rather corresponds to a quantum many-body system in one dimension (quantum Ising chain in a transverse field), which can be solved by the standard onedimensional Jordan-Wigner transformation [5]. However, here it is useful to observe how the "fermion field" is introduced in the path integral (or equivalently the two-dimensional statistical mechanics) viewpoint [6]. First let us introduce the disorder field. The two-point correlation function of the disorder field should measure the "domain wall free energy". Thus it can be defined by the partition function in the presence of a twist (flipping of the sign of the exchange interaction, or equivalently introducing the $\mathbb{Z}_{2}$ gauge field) along a path connecting the two points. It can be easily seen that the choice of the path is arbitrary. Namely, two different choices of the path result in the same partition function, because the path can be deformed by a gauge transformation. The disorder field can then be identified with the endpoint of a "string", namely the flip of the exchange interaction along a path connecting the location to the infinity.

The fermion field $\psi$ is identified with a product of the order parameter (spin) field $\sigma$ and the disorder field $\mu$. When two $\psi$ fields are exchanged, one of the spin field $\sigma$ must cross the string attached to the disorder field which constitutes the other $\psi$. Thus the negative sign follows, as expected for the fermion field (Fig. 1). As already mentioned, this example of the two-dimensional classical Ising model rather corresponds to the original Jordan-Wigner transformation in one spatial dimension. Therefore it does not directly give the desired Jordan-Wigner transformation for quantum systems in two and higher (spatial) dimensions.

Nevertheless, the Jordan-Wigner transformation may be extended to higher dimensions, based on a similar picture. The essence to make the transformation to work in two and higher 


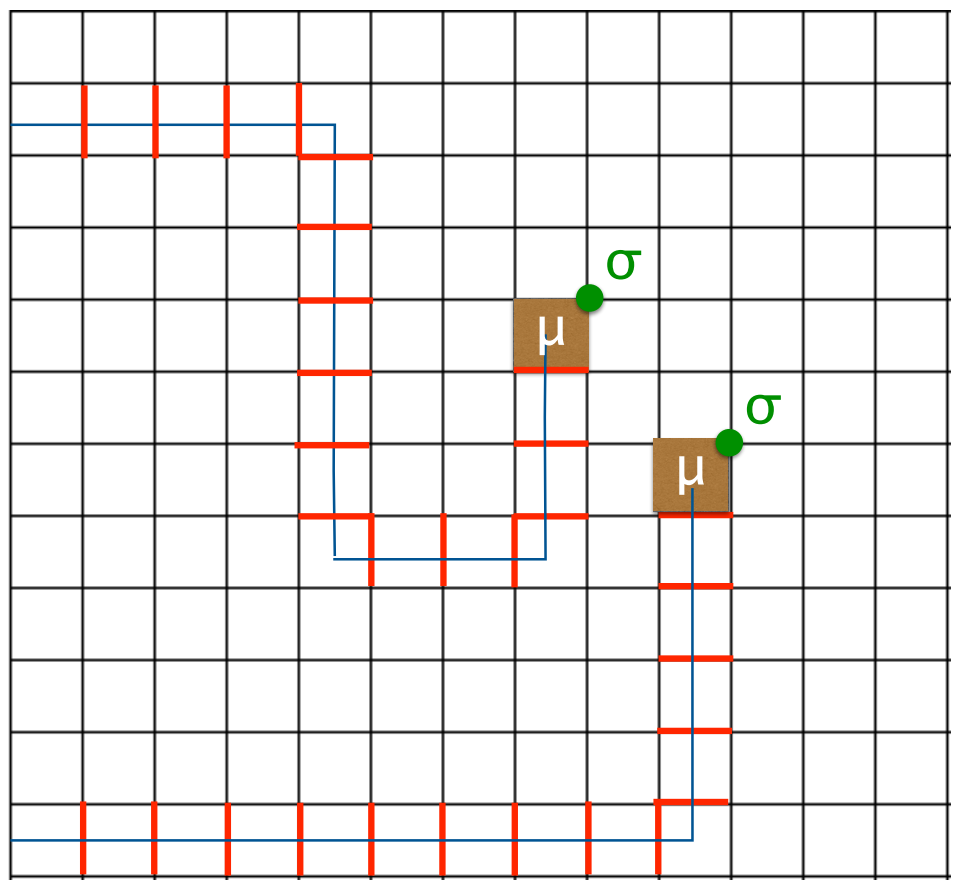

Figure 1: The disorder field $\mu$ and the spin field $\sigma$ in the classical two-dimensional Ising model, following Ref. [6]. Here the Ising model is defined on the square lattice, where an Ising spin is placed on each vertex $\mathbb{r}$. The exchange interaction is present on every link. A string is introduced by flipping every exchange interaction (shown in red) crossing the given path (shown as blue lines). The disorder field $\mu$ is the open end of a string. The fermion field $\psi$ is introduced as a product of the disorder field $\mu$ and the neighboring spin field $\sigma$ with a certain convention (here we chose $\sigma$ on the upper-right corner of the plaquette corresponding to the location of $\mu$ ). The strings can be deformed freely by gauge transformations (redefinition of the local spin variables $\sigma_{\mathbb{I}} \rightarrow-\sigma_{\text {II }}$ ). However, the factor $(-1)$ appears when the spin field $\sigma$ crosses a string. This provides the fermion statistics when the two fermion fields $\psi$ are exchanged. The Jordan-Wigner transformation in two (and higher) dimensions can be introduced in a similar manner, with the string as a quantum mechanical operator which is still deformable thanks to a constraint on the quantum states (see the text). 
dimensions is the "deformability" of the string, namely the equivalence of strings connecting identical pair of points along different paths. In the Hamiltonian formulation of quantum systems, the string should be identified with a quantum mechanical operator localized along the path. Two string operators on different paths are obviously different as operators, and thus the deformability cannot hold as an operator identity. However, the equivalence can be imposed as a constraint on the quantum state. Such a constraint on physical states is a characteristics of quantized gauge theory [7]. Therefore, the deformable string can arise naturally in a lattice gauge model. In fact, the exactly solvable "toric code" [8] in 2 spatial dimensions is related to $\mathbb{Z}_{2}$ gauge theory on the lattice, and contains "electric" and "magnetic" charges as elementary excitations. They appear at the ends of appropriate string operators. Somewhat similarly to the fermion field in the Ising model in $1+1$ dimensions, the fermion operator in the toric code can be identified with the product of the electric and magnetic charges. This implies that fermions can emerge in bosonic quantum spin models. Levin and Wen [9] discussed a more general class of quantum spin models withe emergent topological excitations; in particular, the emergence of fermions from the end of strings is also extended to $3+1$ dimensions (see also [10]).

Fermions as emergent excitations in bosonic systems had been discussed for a long time in the context of fermionic spinons in quantum spin liquids [11]. However, earlier arguments were largely based on uncontrolled approximations. More recent developments discussed above, and also the remarkable exactly solvable honeycomb lattice model by Kitaev [12] theoretically established that the fermions can emerge as elementary excitations in (bosonic) quantum spin systems. The emergent fermions have also become a subject of intense experimental studies [13]. While the Jordan-Wigner transformation was not mentioned in the original paper [12], the Kitaev honeycomb model can be also regarded as an example of a two-dimensional Jordan-Wigner transformation of a free Majorana fermion model.

Obviously, only a special class of bosonic (quantum spin) models exhibit emergent fermions. On the other hand, it turned out for a given fermionic system on a lattice, the mapping to a bosonic quantum spin system maintaining the locality does exist in general $[14,15,16]$. The recent developments in the highlighted papers and their sequels [17, 18, 19, 20, 21] led to various new findings, including the transformations keeping the symmetries manifest, the connection to the higher-form symmetries, the connection to earlier heuristic constructions, and the reduction of the number of auxiliary qubits. While the general construction is quite nontrivial and there are several subtleties, as an illustration I quote the quantum spin model equivalent to the standard spinful Hubbard model on the square lattice, as derived in the highlighted paper 3, in Fig. 2. Perhaps it is appropriate to call such a mapping as JordanWigner transformation in higher dimensions, as it is well-defined for generic fermionic lattice models and is exact.

There are many interesting directions to build upon these advancements. A natural one would be to systematically study quantum spin models which can be mapped to free fermions. Generally speaking, such a model is rather complicated or artificial as a quantum spin system. However, we should recall that Kitaev's honeycomb lattice model looked quite artificial but then was found to be rather naturally realized in the presence of the spin-orbit interaction [22]; there could be exactly solvable nontrivial spin models which are physically important. Some of the models could be also "synthesized" on optical lattices. Even if the exactly solvable model itself cannot be realized, it might give an important clue in deter- 


$$
\begin{aligned}
& -\frac{t}{2} \sum_{\mathbf{r}}\left(-\hat{Y}_{\mathbf{r}}^{(1)} \hat{Y}_{\mathbf{r}}^{(2)} \hat{X}_{\mathbf{r}+\hat{\mathbf{x}}}^{(1)} \hat{X}_{\mathbf{r}+\hat{\mathbf{x}}}^{(2)}+\hat{X}_{\mathbf{r}}^{(1)} \hat{Y}_{\mathbf{r}}^{(2)} \hat{Y}_{\mathbf{r}+\hat{\mathbf{x}}}^{(1)} \hat{X}_{\mathbf{r}+\hat{\mathbf{x}}}^{(2)}+\hat{X}_{\mathbf{r}}^{(2)} \hat{Z}_{\mathbf{r}+\hat{\mathbf{x}}}^{(1)} \hat{X}_{\mathbf{r}+\hat{\mathbf{x}}}^{(2)}+\hat{Z}_{\mathbf{r}}^{(1)} \hat{Y}_{\mathbf{r}}^{(2)} \hat{Y}_{\mathbf{r}+\hat{\mathbf{x}}}^{(2)}\right) \hat{Z}_{\mathbf{r}+\hat{\mathbf{x}}}^{(3)} \\
& -\frac{t}{2} \sum_{\mathbf{r}}^{\prime}\left(\hat{Y}_{\mathbf{r}}^{(1)} \hat{X}_{\mathbf{r}}^{(2)} \hat{X}_{\mathbf{r}+\hat{\mathbf{y}}}^{(1)} \hat{X}_{\mathbf{r}+\hat{\mathbf{y}}}^{(2)}-\hat{X}_{\mathbf{r}}^{(1)} \hat{X}_{\mathbf{r}}^{(2)} \hat{Y}_{\mathbf{r}+\hat{\mathbf{y}}}^{(1)} \hat{X}_{\mathbf{r}+\hat{\mathbf{y}}}^{(2)}+\hat{Y}_{\mathbf{r}}^{(2)} \hat{Z}_{\mathbf{r}+\hat{\mathbf{y}}}^{(1)} \hat{X}_{\mathbf{r}+\hat{\mathbf{y}}}^{(2)}-\hat{Z}_{\mathbf{r}}^{(1)} \hat{X}_{\mathbf{r}}^{(2)} \hat{Y}_{\mathbf{r}+\hat{\mathbf{y}}}^{(2)}\right) \hat{Y}_{\mathbf{r}}^{(3)} \hat{X}_{\mathbf{r}+\hat{\mathbf{y}}}^{(3)} \\
& +\sum_{\mathbf{r}}\left(U-\mu \hat{Z}_{\mathbf{r}}^{(1)}\right) \frac{1+\hat{Z}_{\mathbf{r}}^{(2)}}{2}
\end{aligned}
$$

Figure 2: The explicit spin Hamiltonian obtained by the Jordan-Wigner transformation of the standard spinful Hubbard model on the square lattice, following the highlighted paper 3. $t, U$, and $\mu$ are respectively the nearest-neighbor hopping, the on-site Coulomb interaction, and the chemical potential of the Hubbard model. $X_{\mathbb{T}}^{\alpha}, Y_{\mathbb{\pi}}^{\alpha}, Z_{\mathbb{\pi}}^{\alpha}(\alpha=1,2,3)$ are three sets of the Pauli matrices at each site $\mathbb{r}$. Compared to the original Hubbard model with the 4-dimensional local Hilbert space, this representation has just one extra qubit per site. See the highlighted paper 3 for more details including the constraints. Different mappings of the same model was also given in earlier papers.

mining the phase diagram of the quantum spin system. The Jordan-Wigner transformation would also be useful for quantum simulation of fermionic models. Philosophically, the generality of the Jordan-Wigner transformation might support the view [10] that fermions in our universe are in fact emergent topological excitations in bosonic systems. In any case, there seem to be many possible applications of the transformation, and it would be worth pursuing them which will hopefully lead to deeper understanding of quantum many-body physics.

\section{References}

[1] G. Ortiz, J. E. Gubernatis, E. Knill, and R. Laflamme, Phys. Rev. A 64, 022319 (2001).

[2] P. Jordan and E. Wigner, Zeitschrift für Physik 47, 631 (1928).

[3] E. Lieb, T. Schultz, and D. Mattis, Annals of Physics 16, 407 (1961).

[4] F. Wilczek, Phys. Rev. Lett. 48, 1144 (1982).

[5] J. B. Kogut, Rev. Mod. Phys. 51, 659 (1979).

[6] L. P. Kadanoff and H. Ceva, Phys. Rev. B 3, 3918 (1971).

[7] J. Kogut and L. Susskind, Phys. Rev. D 11, 395 (1975).

[8] A. Kitaev, Annals of Physics 303, 2 (2003).

[9] M. A. Levin and X.-G. Wen, Phys. Rev. B 71, 045110 (2005).

[10] X.-G. Wen, Quantum field theory of many-body systems: from the origin of sound to an origin of light and electrons (Oxford University Press, Oxford, 2007). 
[11] G. Baskaran, Z. Zou, and P. Anderson, Solid State Communications 63, 973 (1987).

[12] A. Kitaev, Annals of Physics 321, 2 (2006), January Special Issue.

[13] Y. Kasahara et al., Nature 559, 227 (2018).

[14] S. B. Bravyi and A. Y. Kitaev, Annals of Physics 298, 210 (2002).

[15] R. C. Ball, Phys. Rev. Lett. 95, 176407 (2005).

[16] F. Verstraete and J. I. Cirac, Journal of Statistical Mechanics: Theory and Experiment 2005, P09012 (2005).

[17] Y.-A. Chen and A. Kapustin, Phys. Rev. B 100, 245127 (2019).

[18] Y.-A. Chen, Phys. Rev. Research 2, 033527 (2020).

[19] A. Bochniak and B. Ruba, Journal of High Energy Physics 2020, 118 (2020).

[20] A. Bochniak, B. Ruba, and J. Wosiek, (2021), arXiv:2107.06335.

[21] K. Li and H. C. Po, (2021), arXiv:2107.14083.

[22] G. Jackeli and G. Khaliullin, Phys. Rev. Lett. 102, 017205 (2009). 